\title{
La comunicación como categoría básica de lo social: una aproximación a la Escuela de pensamiento social de la Universidad de Chicago
}

\section{Communication as a Main Social Category: An Approach to the Chi- cago School of Social Thought}

\author{
Manuel Martín Algarra, catedrático de la Universidad de Navarra. \\ Mariano Navarro Arroyo, investigador de la Universidad de Navarra
}

Recibido: 29-X-2008 - Aceptado: 10-XI-2008

Resumen:

Desde el primer tercio del siglo XX las aproximaciones al fenómeno de la comunicación se efectuaron bajo el paradigma de la comunicación de masas, lo que, si bien dotó a los estudios en comunicación de una base empírica sólida que daba razón de hechos puntuales, alejó la posibilidad de contar con una base conceptual comprensiva que diera cohesión a los avances empíricos en el contexto amplio de las ciencias sociales. Pero con anterioridad a las aproximaciones empíricas de la investigación en comunicación de masas hubo un esfuerzo teórico coherente que identificó a la comunicación como una categoría social básica y fundamental en la formación del individuo y en la dinámica social: el pensamiento social de la Escuela de Chicago. Los frutos de dicha reflexión no han sido explorados en su totalidad por la teoría de la comunicación, y su recuperación permite augurar una mejor comprensión de la comunicación como un fenómeno central en la formación y el devenir de la sociedad.

Palabras clave:

Comunicación de masas, Escuela de Chicago, comunidad, pragmatismo, participación, liberalismo.

Abstrac:

Ever since the first third of the twentieth century most of the approaches toward the study of communication took place under the mass communication paradigm, which, on one hand, gave communication studies a solid empirical base that explained specific phenomena, but, at the same time, withered away the hopes of ever gaining a solid conceptual comprehensive base that coherently placed those empirical conclusions in the wider context of social science. But prior to those empirical approaches to mass communication study a coherent theoretical effort which identified communication as a basic and fundamental social category took place: it was the social thought of the Chicago School. The results of such theoretical corpus have not been fully explored by communication theory, and their careful examination invites to hope for a better comprehension of communication as a central phenomenon in both the formation and development of society.

Key words:

Mass Communications, Chicago School, community, pragmatism, participation, liberalism. 


\section{Introducción}

La irrupción en el primer tercio del siglo XX de la mass communication research (MCR) como aproximación de naturaleza empírica al fenómeno de la comunicación pública tuvo consecuencias contradictorias. Por una parte desarrolló notablemente la investigación sobre aspectos puntuales del fenómeno, demostrando la relevancia de la comunicación mediática en la vida social y la pujanza del nuevo campo de estudio como motor de la investigación social empírica. Pero por otro lado, alejó la investigación en comunicación de la conceptualización, de la búsqueda de sus axiomas, de sus fuentes normativas y de la reflexión sobre los fundamentos teóricos de la comunicación como categoría básica.

En los últimos años, la investigación de la comunicación en España ha continuado buscando su fundamentación bien en la investigación administrativa inspirada por Lazarsfeld y los demás "padres fundadores de la MCR”, bien en la crítica, inspirada en la Escuela de Frankfurt, bien en los desarrollos posteriores de ambas. Con alguna excepción (Berganza, 1999 y 2000; Sánchez de la Yncera, 1994), la teoría de la comunicación española no ha prestado especial atención a las aportaciones a este campo de la escuela de pensamiento social de Chicago.

Con anterioridad a la explosión de investigaciones y publicaciones de carácter empírico en el dominio de la sociología de la comunicación -entendida en sentido amplio-, hubo una fértil reflexión académica sobre la realidad de la comunicación y su carácter fundante de la vida social. La investigación empírica en nuestro campo, sumamente floreciente a partir de los años 30 del siglo pasado, eclipsó esa reflexión. Quizás por ello, los investigadores empíricos han manifestado con frecuencia la necesidad de desarrollar una reflexión teórica que dé razón de sus propios hallazgos y permita integrarlos de un modo coherente.

El problema no deriva del enfoque metodológico empírico adoptado para investigar la realidad de la comunicación, sino más bien de la renuncia -más o menos explícita, más o menos consciente- de este tipo de investigación a realizar exploraciones y propuestas de carácter conceptual y normativo sobre la comunicación que den razón tanto del fenómeno como de su situación en un contexto humano y social amplio. Como veremos, la obra de Lippmann sirve para dar razón del "objetivismo” y del "neutralismo" de la MCR. No obstante, la historia de la investigación de la comunicación cuenta con una posición diferente que, sin hacer ninguna renuncia metodológica, sugiere la necesidad de una ciencia social meliorativa: tal es el caso de la Escuela de pensamiento social de la Universidad de Chicago, que aporta un pensamiento original y fecundo sobre la comunicación. 


\section{La historia "oficial" de la investigación en comunicación}

Carey (1996) sostiene que existe lo que podríamos llamar una "historia oficial" de la investigación en comunicación, asumida en líneas generales por todos, y que ha pasado a formar parte de la cultura común de nuestro campo de estudio. Esa historia comienza en la I Guerra Mundial y distingue las tres consabidas etapas según la percepción académica del nivel de influencia de los medios a lo largo del tiempo: etapa de los efectos poderosos, la de los efectos limitados y la de la sociedad de masas.

En la primera de ellas, la de los efectos poderosos, se aplican las ideas científicas de moda en el momento, a saber; el determinismo darwinista. A esa idea responde la aplicación masiva de la propaganda en la Guerra y las cautelas que frente a ella se tomaron -especialmente en los Estados Unidos- ya en la Guerra y en la inmediata postguerra. Hay autores que se han encargado de destacar que es precisamente esa idea del poder de los medios la que configura el desarrollo de la práctica de la comunicación entendida como propaganda para los tiempos de paz, que tan bien encarnada queda en la figura de Edward Bernays y descrita en sus obras. Esta etapa de la historia convencional de la investigación en comunicación nos ha dejado, casi a modo de icono, el caso de Orson Welles y su “Guerra de los mundos", que proporcionó la que tal vez sea la primera evidencia empírica sistemáticamente estudiada de los efectos poderosos e inmediatos de los medios de comunicación de masas y a la que se dio el nombre de teoría de la aguja hipodérmica.

La propia dinámica de la investigación empírica, que implica la revisión sistemática de sus métodos y resultados, propició la segunda etapa recogida en la historia convencional de la investigación en comunicación, la de los efectos moderados. La continuación, en el periodo de entreguerras, del estudio sistemático de los efectos de los medios llevó a importantes centros académicos como Chicago o Columbia y al propio Departamento de Guerra del Gobierno de los Estados Unidos a dedicar muchos recursos a este tipo de investigación. Las conclusiones a las que llegaron estos estudios confirmaron que no había evidencias empíricas que demostrasen que los contenidos o intenciones de los medios fueran acompañados de efectos poderosos en las audiencias. Aunque tendemos a relacionar a los "Padres Fundadores de la investigación en comunicación” con el estudio de los efectos de los medios, pocas veces se tiene en cuenta que fue precisamente la investigación que ellos desarrollaron la que puso las bases de la idea central de esta segunda fase de la historia convencional de la investigación en comunicación. La investigaciones del equipo de Hovland en Yale (1949, 1953), o las de Lazarsfeld y sus colaboradores publicadas en The People's Choice (1944) y Personal Influence (1955), por ejemplo, muestran que hay mecanismos psicológicos de defensa frente al impacto de la propaganda y de los medios en general. Esta conclusión fue señalada por Joseph Klapper ya a finales de los años 40 y completamente articulada, también desde el punto de vista estadístico y de la evi- 
dencia empírica, en la obra que resume esta segunda etapa de la historia convencional de la investigación en comunicación: The Effects of Mass Communication (1960). En ella, Klapper concluye que el temor a la propaganda o a la manipulación a través de los medios, que influyó de manera tan decisiva en la vida cotidiana de los Estados Unidos en el primer tercio del siglo XX, carecían de fundamento y eran una reacción casi histérica. La investigación empírica había demostrado que los medios de comunicación de masas sólo tienen efectos sobre los individuos cuando refuerzan sus propias actitudes o convicciones, y muy raramente, si es que alguna vez en absoluto, en sentido contrario. Los medios, por tanto, no podían ser percibidos como la fuente de la revolución contra el sistema democrático, sino más bien un vehículo que refuerza la cultura establecida y que refuerza el status quo. Con tal conclusión bien establecida, apoyados en las ideas de las teorías psicológicas de la consistencia (Heider, 1948, 1958), la investigación empírica avanza por la línea de los usos y gratificaciones (Festinger, 1957; Katz y Foulkes, 1962) y el interés varía desde lo que los medios hacen con la gente hacia lo que la gente hace con los medios.

La tercera etapa de esta "historia oficial" mencionada por Carey es la de la teoría de la sociedad de masas. El sociólogo Daniel Bell (1961) la describe como el periodo en el que la revolución del transporte y las comunicaciones permite a cada individuo una completa autonomía, pero que al mismo tiempo, paradójicamente, produce el fenómeno del aislamiento social. Ese individualismo potenciado por la tecnología permite acabar con los vínculos que venían definiendo la identidad del hombre hasta ese momento. Desaparece el sentido de necesidad de los viejos grupos primarios como la familia o la comunidad local, a los que se impone la libertad individual. La fe de las antiguas comunidades de creyentes, otra de las clásicas formas de identidad, es puesta en duda, e igualmente desaparecen las referencias objetivas sobre la moral o el gusto, que pasan a ser ideas en constante cambio. Así, las relaciones entre los individuos dejan de ser algo orgánico para convertirse en una realidad meramente tangencial. "A causa de todo esto, el individuo pierde un sentido coherente de su propio ser. Sus ansiedades aumentan. Y de aquí resulta la búsqueda de nuevas creencias. La situación está por tanto preparada para la llegada de un nuevo líder carismático, un mesías secular que, otorgando a cada persona la gracia necesaria y la plenitud de personalidad, proporcione un sustituto para el orden destruido por la sociedad de masas" (Bell, 1961: 22). Los medios de comunicación, en esta tercera etapa de la historia estándar de la comunicación, destruyen los viejos valores éticos y estéticos que se imponían al hombre moderno pero, al mismo tiempo, cargan sobre ese mismo hombre otros valores que lo dominan. Ha desaparecido el orden tradicional pero ha sido recreado uno nuevo en el mercado, la política de masas y el consumismo.

Como señala Carey, ésta es la "historia oficial” de la investigación en comunicación, la asumida por la comunidad académica desde que se puede hablar de la existencia de tal comunidad. Son diversas las historias 
del campo escritas desde sus comienzos, pero, en mayor o menor medida, todas recogen esas etapas (WahlJorgensen, 2000). Ciertamente hay visiones más centradas en la medición de los efectos y otras que se ciñen más a la crítica del resultado de tales efectos pero, en última instancia, se acepta ese esquema de la historia que tiene que ver con la influencia de los medios en el sistema social.

\section{Insuficiencias del liberalismo antropológico (y comunicativo)}

A pesar de ello, el propio Carey afirma que, aunque no hay en el siglo XIX nada que pueda ser llamado con propiedad investigación en la comunicación de masas, sin embargo, la historia convencional no se entiende bien sin tener en cuenta dos corrientes de gran importancia en la configuración de la reflexión sobre los medios de comunicación: en primer lugar el Liberalismo y después la Escuela de Pensamiento Social de la Universidad de Chicago. La época considerada en la "historia oficial” no es más que el punto de conexión entre ambas y, en última instancia, la salida más neutra posible al problema intelectual de la posibilidad de un orden social verdaderamente democrático basado en la libertad de expresión y en la opinión pública.

El liberalismo parte de una antropología individualista en la que acaban manifestándose las contradicciones entre la libertad y la naturaleza, entre la radical condición individual del ser humano y la imposibilidad de desplegarla fuera del contexto social. Dejando de lado las cuestiones filosóficas y antropológicas de fondo, a pesar de que no son en absoluto ajenas a la teoría de la comunicación, parece claro que las revoluciones antiliberales de los siglos XIX y XX tienen que ver con el rechazo a una noción que entiende al hombre sólo como un individuo que puede imponerse de manera absoluta a los demás en virtud de su fuerza, su inteligencia, su herencia, o su suerte.

El liberalismo inspira una teoría de la comunicación a partir de los principios que inspiran el libre mercado. Su concepto básico, el de libre mercado de las ideas, se nutre en Milton y Mill. Al ocuparse de la comunicación, el liberalismo se ha entendido de dos maneras complementarias. La primera sostiene que la comunicación opera sobre la base de la búsqueda de la verdad a través de la libertad de expresión. Cualquier idea, por tanto, puede y debe ser expresada en la palestra pública puesto que de la libre confrontación de ideas surgirá la verdad, de la misma manera que de la libre oferta de productos en el mercado surge el mejor precio y la mejor calidad. La segunda manera de entender la comunicación en el liberalismo parte, no de la búsqueda de la verdad, que se considera inalcanzable o indeterminable, sino de la persecución del interés individual, lo que se concreta igualmente en ausencia de límites a la libre expresión. En este caso el objetivo no es la verdad, que tiene una dimensión pública, sino la perfección de la información que permite elegir más eficazmente lo que cada individuo desea. Ésa es la mejor racionalidad: la de encontrar los me- 
jores medios para los propios fines individuales. En ambos casos -la búsqueda de la verdad y la búsqueda del interés- la misma "mano oculta" que actúa en el mercado permitirá que se alcance el equilibrio.

El liberalismo basado en los derechos comprendió la libertad como la autonomía individualista, poniendo en tela de juicio la bondad de la influencia que pudieran ejercen sobre los individuos las distintas formas de socialización. De esta manera se fue gestando un paradigma, muy difundido, según el cual existe una oposición natural entre el individuo y la sociedad, y así lo percibió John Dewey, inspirador de la Escuela de Chicago (Savage, 2002). La consideración de la teoría de la comunicación desde el concepto liberal de libertad, en última instancia, da razón del colapso del sistema y de las insuficiencias que necesariamente exhibe cualquier tipo de comunicación ejercida desde la comprensión liberal del mundo y las personas. Esta tensión entre una comprensión liberal a ultranza de la comunicación fue lo que propició el debate que Dewey sostuvo con uno de los más clarividentes teóricos políticos de su tiempo, Walter Lippmann.

Walter Lippmann ofrece en Public Opinion (1922) una descripción del colapso del sistema liberal en lo que se refiere a la comunicación pública. En sus páginas elabora y conceptualiza sus experiencias en París y Versalles como asesor del Presidente Woodrow Wilson tras la I Guerra Mundial. En ese contexto histórico toman cuerpo las ideas de Lippmann sobre la imposibilidad de un sistema político verdaderamente basado en la libertad de expresión y en la opinión pública. Sostiene que el ciudadano medio carece de competencia para conocer adecuadamente las cuestiones que se refieren al gobierno de los asuntos públicos. Más aún: la mayor parte de los ciudadanos ni siquiera están interesados en tales asuntos. A ello se une que los medios no ofrecen una visión comprensiva de la realidad que permita a los votantes tomar decisiones adecuadas acerca de cuestiones de la política. El diagnóstico de Lippmann resulta fundamental para explicar por qué la investigación sobre la comunicación, y especialmente sobre los medios de comunicación de masas, se acaba centrando en esa época en la pura medición de sus efectos, sin realizar juicios "normativos". Existe una incompetencia psicológica de la gente para participar en la vida pública y por eso Lippmann propone en última instancia un gobierno de científicos, de sabios y expertos en los diversos temas, de gente preparada y dedicada profesionalmente a las cuestiones que afectan a la vida pública que el ciudadano común no puede, y en muchos casos no le interesa, conocer. La pretendida neutralidad, positivamente buscada, de la investigación funcionalista encuentra aquí su nexo con la tradición liberal: la investigación en comunicación es exclusivamente investigación sobre los efectos de los medios. Cualquier intento normativo sería pretencioso y, cuando menos, poco científico. El diagnóstico de Lippmann se basa en el papel político que la comunicación pública tiene en los sistemas liberales. Ciertamente, desde ese punto de vista, el colapso de la comunicación, como el que describe Lippmann, es el colapso de la democracia. 


\section{Alternativas comunicativas y comunitarias: Chicago y su pensamiento social}

Pero cabe otorgar otro papel más amplio a la comunicación. Es lo que hace John Dewey cuando afirma que no es que la comunicación se dé en la vida social sino que la sociedad se da en la comunicación. Tras esa afirmación hay una idea de que la vida humana sólo se entiende en la comunidad: no es por tanto una lucha entre individuos, como proponían los modelos de las ciencias sociales, inspirados en el evolucionismo darwiniano, como el de Herbert Spencer entonces tan difundido. Dewey creía que la plenitud humana sólo se puede alcanzar en compañía de los otros, de ahí que a lo largo de los años su ámbito de preocupaciones intelectuales girara desde la filosofía hacia la política, la educación y la comunicación, de manera notable a partir de su incorporación a la Universidad de Michigan y posteriormente a la de Chicago.

La ciudad de Chicago experimentó un inmenso crecimiento en los últimos años del siglo XIX. A comienzos del siglo XX era, por tanto, una ciudad de aluvión, a la que llegaban inmigrantes de todo el mundo y muy poco parecida a la Nueva Inglaterra de un siglo antes que había dado origen a los Estados Unidos. En 1900, por ejemplo, habían nacido en Chicago sólo la mitad de sus 1,7 millones de habitantes, que a su vez eran más del doble de los que poblaban la ciudad unos quince años antes. Convivían en ella una impresionante riqueza económica, capaz de atraer masas de población de todo el mundo, y una igualmente impresionante miseria fruto de la explotación, del desarraigo y también de la corrupción.

En 1890 un grupo de miembros de iglesia Baptista fundaron la Universidad de Chicago financiados por el magnate del petróleo John D. Rockefeller. La joven universidad adoptó como modelo los grandes centros académicos alemanes y pronto contó con un brillante plantel de profesores. Así, la de Chicago fue la primera universidad de los Estados Unidos en crear un Departamento de Sociología, que fundó y dirigió en sus primeros años Albion Small. Una idea básica de Small era que el Departamento de Sociología se ocupara de los problemas reales y Chicago en ese momento era un laboratorio de excepción para el estudio de los problemas urbanos y sociales: la investigación social debía estudiar cómo se ajustaba la democracia americana, rural y comunitaria, forjada en pequeñas comunidades relativamente homogéneas, a una situación tan radicalmente distinta a la original, como era la de la ciudad de Chicago en los primeros años del siglo XX.

Georg Simmel fue el autor europeo que más influyó en la sociología de Chicago. Albion Small tradujo varias obras suyas que se publicaron en el American Journal of Sociology que había comenzado a publicarse en el Departamento de Sociología de Chicago, y Park estudió con él en Berlín. Más tarde, el mismo Park escribiría un manual de introducción a la sociología lleno de influencia simmeliana con el que se formarían muchas generaciones de sociólogos en Estados Unidos. Frente a Durkheim, que se apoyaba en el análisis empírico de la realidad, o aWeber, que enfocaba el análisis sociológico alrededor de conceptos más complejos, Simmel consideraba que 
la sociología debía avanzar por medio de la comprensión del problema de la socialización. Esta preocupación por la constitución misma de la sociedad y la realidad social está en la base de las ideas más características de la Escuela de Chicago en lo que se refiere a la comunicación (Levine, Carter y Gorman, 1976a y 1976b):

- $\quad$ La sociedad consiste en la comunicación entre los individuos.

- Toda comunicación humana representa algún tipo de intercambio que tiene efectos recíprocos sobre quienes participan en ella.

- $\quad$ La comunicación se da entre individuos que se encuentran en niveles de diversa distancia social.

- La comunicación humana satisface ciertas necesidades básicas, como la compañía, persigue beneficios como la educación, etc.

- Ciertos tipos de comunicación se convierten en estables y quedan fijados con el tiempo en la estructura social.

Además de la influencia europea, hay otras características comunes a Dewey, Cooley, Park y Mead, los autores más característicos de esta escuela de pensamiento social (Rogers, 1994: 137-202). Todos ellos nacen a mediados del siglo XIX, en torno a la Guerra Civil, eran protestantes y concebían la sociología como una ciencia meliorativa, esto es para acometer la solución de los problemas sociales y no como mero instrumento de observación. Los autores de la Escuela de Chicago se reúnen en torno a John Dewey, primero en la Universidad de Michigan, donde fue profesor de Cooley y Park y, más tarde, con la excepción de Cooley que permaneció en Ann Arbor, en la de Chicago, a la que se incorporarían Mead y Park.

Hans Joas define la Escuela de Chicago como "la combinación de una filosofía pragmática, de un intento de dar una orientación política reformista a las posibilidades de la democracia en condiciones de rápida industrialización y urbanización, y de los esfuerzos por convertir la sociología en una ciencia empírica concediendo gran importancia a las fuentes precientíficas del conocimiento empírico" (Joas, 1990: 23). Un breve análisis de las notas de la definición puede alumbrar el camino.

En efecto, el enfoque pragmatista (más o menos marcado, pero siempre presente) en los miembros de la Escuela de Chicago supone una manera concreta de aproximarse a las cuestiones que abordan. El pragmatismo, iniciado por Peirce y James, concebía el conocimiento como una búsqueda sin un resultado definitivo e inamovible y que responde a un propósito o interés concreto. Los pensadores de la escuela de Chicago se encuentran con una situación problemática a la que buscan dar una respuesta: la nueva forma de vida industrializada en los núcleos urbanos supone un reto a la democracia tradicional, a la democracia articulada 
en torno a pequeñas comunidades. Y su orientación reformista -o meliorista- les llevó a indagar las maneras en las que lo valioso del orden comunitario tradicional y rural podría recrearse en el nuevo contexto urbano e industrial. Este es el propósito último de John Dewey: conseguir el paso de "la gran sociedad" a "la gran comunidad”. Y es a través de este prisma que Dewey analiza lo esencial de la vida en común, para concluir que la comunidad y comunicación tienen una relación recíproca muy profunda.

Cooley influyó en Mead y ambos son considerados fundadores del interaccionismo simbólico. Frente a las posturas más biologicistas de moda en la época, negaban que los instintos fueran la base de la personalidad humana y pensaban que ésta quedaba configurada más bien por la relación interpersonal. Desde el punto de vista metodológico, los autores de Chicago practicaban en mayor o menor medida una sociología de corte empírico: cualquier problema social exigía para su solución un profundo conocimiento y un estudio minucioso con métodos etnográficos como la observación y las entrevistas en profundidad así como el uso indicadores sociales de naturaleza cuantitativa. Practicaban una investigación social aplicada a problemas reales, alejándose así de las grandes visiones organicistas de la vida social herencia del darwinismo social. Park, por su parte, fue periodista antes de marcharse a Alemania a hacer su tesis doctoral. También se unió al grupo el periodista Franklin Ford que había fundado el periódico Thought News. Ford daba una gran importancia al papel de la prensa en la vida social y quería que el periódico ofreciera una información precisa y objetiva que permitiera a los lectores tomar decisiones sobre los asuntos públicos con pleno conocimiento. Su proyecto era hacer un periódico que fuera una mezcla de journal académico y banco de datos. Como cabe esperar, sólo vio la luz un número, pero la experiencia produjo a Dewey un gran impacto. Es posible que Thought News explique en parte la inclinación del grupo a considerar la prensa como una fuente de integración social para una sociedad desarraigada como la del Chicago de comienzos del siglo XX.

La prensa, junto con la educación, era la única vía para reconstruir en las grandes zonas urbanas e industriales las comunidades en las que había visto la luz la democracia americana. "Sólo la comunicación puede crear una gran comunidad”, escribe Dewey en The Public and its Problems (1927). En esta obra afirma que la sociedad urbana de masas difícilmente puede organizarse según los principios inspiradores de la democracia americana si no restauraban los modos de funcionamiento de las pequeñas comunidades rurales en las que esa democracia surgió. Los medios de comunicación y transporte que se generalizaban en esa época podrían ayudar a gentes llegadas de lugares muy distintos, con culturas, orígenes sociales e incluso idiomas diferentes, a conectar unos con otros y dejar de ser un agregado amorfo de individuos desarraigados para convertirse en una gran comunidad. La comunicación pública -la negociación, la información, el debate-, a falta de una cultura y tradición comunes, era el único medio por el que podrían organizarse y mantenerse unidas las nue- 
vas comunidades urbanas surgidas en los Estados Unidos. Frente a las ideas puramente liberales, la Escuela de Chicago sostiene que el orden social no se hereda ni se alcanza inconscientemente, sino que se forja cuando gente diferente se une para crear una cultura común y para estructurar esa cultura en instituciones sociales. La comunicación, por tanto, no es fundamentalmente un ejercicio individual y solitario como sostiene el liberalismo, sino una fuente de cohesión social, de integración. En este contexto se entienden las conocidas palabras de Dewey: "La sociedad existe no sólo por la transmisión, la comunicación, sino que sería más apropiado decir que la sociedad existe en la transmisión, en la comunicación” (Dewey, 1916: 5).

\section{Balance: lo que cabe esperar del pensamiento social de Chicago en la teoría de la comunicación}

Como señala Carey, la historia "oficial" de la investigación en comunicación se queda reducida a la "ciencia” neutra de la MCR. El utilitarismo liberal y el pensamiento social de la Escuela de Chicago son pocas veces vinculados al mainstream de la investigación en comunicación. Sin embargo, las ideas vertidas en esas dos tradiciones, habitualmente dejadas al margen de la historia convencional, arrojan luces muy necesarias para comprender la verdadera importancia de la investigación en comunicación.

La Escuela de Pensamiento Social de Chicago precede al comienzo de la "historia oficial" de la investigación en comunicación y ofrece una salida distinta a la que el funcionalismo da al colapso de la teoría liberal de la comunicación diagnosticado porWalter Lippmann. Parte de la base de que la comunicación no es un fenómeno social más. No es algo que se da en la vida social sino que está en su mismo origen. Sin dejar de reconocer la importancia de los grupos primarios en la formación del tejido social, en el proceso de urbanización que se da en la transición del siglo XX al XX en los Estados Unidos, los autores de Chicago, especialmente Dewey, vieron en los medios de comunicación una vía para recrear las condiciones de la vida pública de esas pequeñas comunidades en las que se forjó la democracia americana. Como señala Carey (1996: 336) para Dewey “la comunicación era un principio ético. Cualquier cosa que impidiera compartir, ampliar y expandir la propia experiencia era un obstáculo que tenía que ser superado, pues aprendemos unos de los otros, tanto de lo que nos hace distintos como de lo que nos hace semejantes. Los nuevos medios ofrecían una oportunidad sin precedentes para ampliar el espacio para el aprendizaje, y para incrementar la capacidad de aceptar y trascender lo particular, de unirse a una comunidad de ciudadanos más amplia sin que ello supusiera sacrificar la propia identidad como miembros de formaciones sociales particulares". Carey considera ingenuo ese optimismo de los autores de Chicago y cifra en él la entrada con tanta fuerza en el campo de estudio de los medios de comunicación del análisis crítico procedente del marxismo y de la ciencia social positivista centrada en los efectos que encarna el funcionalismo. Sin 
embargo, como el mismo Carey señala también, las ideas de la Escuela de Chicago se han mantenido en el tiempo y ahora vuelven con fuerza inspirando investigaciones muy diversas - por ejemplo, en la teoría de los públicos (Míguez, 2006) - en el campo de la comunicación.

Tal como se ha visto, la teoría de la comunicación que se desprende de la reflexión de la Escuela de Chicago apunta en una dirección más humanística y conceptual que la emprendida por la mass communication research, y los frutos que de ella puedan desprenderse contribuirán a clarificar la relación de reciprocidad que se establece entre el individuo y la comunidad en la que actúa y se desarrolla. Así pues, la idea misma de comunicación sugiere una sociedad abierta en la que la participación en la vida pública juega el doble papel tanto de ideal como de condición de posibilidad. Dicha comprensión parece ser un marco especulativo adecuado para el examen de una realidad -la comunicación- que tiene muchas maneras de concretarse, tanto naturales como mediadas.

Por otro lado, debido al peso específico de los pensadores de la Escuela de Chicago y al papel de preeminencia que tuvieron durante algunos años en el ambiente académico norteamericano, su estudio detallado en el campo de la teoría de la comunicación podrá contribuir a una mejor comprensión de esa historia "oficial” de la investigación en comunicación a la que ha contribuido de manera relevante a pesar del tímido o negligente reconocimiento recibido.

\section{Referencias bibliográficas}

Bell, D. (1961): The End of Ideology, Nueva York: Collier.

Berganza Conde, M. R. (1999): “Hacia una recuperación del pensamiento de los pioneros: el concepto de comunicación en la teoría sociológica de Robert E. Park”, Comunicación y Sociedad, vol. 12, pp. 49-75.

- (2000): Comunicación, opinión pública y prensa en la sociología de Robert E. Park, Madrid: CIS, Siglo XXI.

Carey, J. W. (1996): “The Chicago School and Mass Communication Research”, en Dennis, E. D.; Wartella E. (eds.): American Communication Research: The Remembered History. Mahwah: Erlbaum, pp. 21-38.

Dewey, J. (1916): Democracy and Education, Nueva York: MacMillan.

- (1927): The Public and its Problems, Nueva York: Holt.

Festinger, L. (1957): A Theory of Cognitive Dissonance, Stanford: Stanford University Press.

Heider, F. (1946): “Attitudes and Cognitive Organization”, Journal of Psychology, vol. 21, pp. 107-112.

- (1958): The Psychology of Interpersonal Relations, Nueva York: Wiley. 
Hovland, C. I.; Janis, I. L.; Kelley, H. H. (1953): Communication and Persuasion: Psychological Studies of Opinion Change, New Haven: Yale University Press.

Hovland, C. I.; Lumsdaine, A. A.; Sheffield, F. D. (1949): Experiments on Mass Communication, Princeton: Princeton University Press.

Joas, H. (1990): “Interaccionismo Simbólico” en Giddens; Turner; et al. La teoría social hoy, Madrid: Alianza.

Katz, E.; Foulkes, D. (1962): “On the Use of Media as «Escape»: Clarification of a Concept”, Public Opinion Quarterly, vol. 26, pp. 377-388.

Katz, E.; Lazarsfeld, P. F. (1955): Personal Influence. The Part Played by People in the Flow of Mass Communications, Glencoe: The Free Press.

Klapper, J. T. (1960): The Effects of Mass Communication, Nueva York: The Free Press.

Lazarsfeld, P. F; Berelson, B.; Gaudet H. (1944): The People's Choice: How the Voter Makes up His Mind in a Presidential Campaign, Nueva York: Duell, Sloan and Pearce.

Levine, D.; Carter, E. B.; Gorman, E. M. (1976): “Simmel's Influence in American Sociology I”, The American Journal of Sociology, vol. 81, pp. 813-845 y pp. 1112-1132.

Lippmann, W. (1922): Public Opinion, Nueva York: Harcourt, Brace and Company.

Míguez González, M. I. (2006): Los públicos en las relaciones públicas: estudio recopilatorio y crítico acerca de las principales aportaciones sobre la investigación de los públicos en las relaciones públicas, Tesis doctoral, Pontevedra: Universidad de Vigo.

Peters, J. D. (1994): “Genealogical Notes on 'the Field’”, en Levy M.; Gurevitch, M. (eds.): Defining Media Studies: Reflections on the Future of the Field. Nueva York: Oxford University Press, pp. 374-381.

Rogers, E. M. (1994): A History of Communication Study. A Bibliographical Approach, Nueva York: The Free Press.

Sanchez de la Yncera, I. (1994): La mirada reflexiva de G. H. Mead: sobre la socialidad y la comunicación, Madrid: CIS, Siglo XXI.

Savage, D. (2002): John Dewey's Liberalism, Carbondale and Edwardsville: Southern Illinois University Press.

Siebert, F. S.; Peterson, T. B.; Schramm, W. (1963): Four Theories of the Press: The Authoritarian, Libertarian, Social Responsibility and Soviet Communist Concepts of what the Press Should Be and Do, Urbana: Illinois University Press.

Wahl-Jorgensen, K. (2000): "Rebellion and Ritual in Disciplinary Histories of U. S. Mass Communication Study: Looking for «The Reflexive Turn»”, Mass Communication \& Society, vol. 3, pp. 87-115. 